\title{
Liquid hydrogen at the thermodynamic conditions of room temperature and a pressure of $490 \mathrm{GPa}$
}

\author{
RANBER SINGH \\ Department of Physics, Sri Guru Gobind Singh College, Sector 26, Chandigarh 160019, India \\ ranber14@gmail.com
}

MS received 14 February 2018; accepted 28 May 2018; published online 12 January 2019

\begin{abstract}
We investigate the effect of nuclear quantum effects in liquid hydrogen at the thermodynamic conditions of room temperature and a pressure of $490 \mathrm{GPa}$. The calculated pair-correlation function shows that the nuclear quantum effects are quite significant for the correct description of liquid atomic hydrogen at high pressures.
\end{abstract}

Keywords. Liquid hydrogen; molecular dynamics; pair-correlation function.

\section{Introduction}

Hydrogen is the simplest possible atom composed of one proton in the nucleus and a single electron orbiting the nucleus. It is the most abundant element in the universe. Hydrogen bonding is very important in many chemical processes. For instance, hydrogen bonding is responsible for water's unique properties, holds complementary strands of DNA together, is responsible for determining the threedimensional structure of folded proteins and so forth. The two-level excitations play an important role in the quantum diffusion of hydrogen in solids at low temperatures [1]. The hydrogen in hydrogenated amorphous silicon makes it to behave like a hydrogen glass [2]. The diffusion of hydrogen in aluminium and its alloys changed its properties [3]. The link between hydrogen embrittlement and stress corrosion cracking in these aluminium alloys has also been discussed. The pressure-dependent behaviour of hydrogen in hydrogen storage materials has been presented in literature [4]. Nanometric carbon CMK-3 modified with $\mathrm{TiO}_{2}$ in anatase phase has been demonstrated as a good candidate for hydrogen storage [5].

On the contrary, the phase diagram of pure hydrogen under pressure is least understood and is an unsolved problem till date. Theoretical and experimental observations indicate that hydrogen would become metallic at high densities [6-8]. However, it is not yet clear at what density the transition from insulating state to a metallic state would occur. At low densities, the $\mathrm{H}$ atoms of different molecules are sufficiently far apart from each other, such that all possible atomic configurations are insulating. At high enough densities, the $\mathrm{H}$ atoms are expected to become sufficiently closer so that hoping of electrons from one atom to another becomes easier with increasing density. Eventually, at extremely high densities the hydrogen system is expected to become metallic. The existence of the metallic state in dense hydrogen under pressure is one of the fundamental questions in high-pressure physics. It has been the elusive Holy Grail of high-pressure physics for many years since its first prediction in 1935 by Wigner and Huntington [9].

Metallic hydrogen has been experimentally realized in the liquid phase at high pressure of about $140 \mathrm{GPa}$ and temperature of about $3000 \mathrm{~K}$ [7]. However, it is still controversial whether this metallic state is molecular, atomic or mixed. The onset of the metallic state in hydrogen under pressure has also been observed at about $270 \mathrm{GPa}$ and $300 \mathrm{~K}[10,11]$. Initially, it was speculated to be due to metallic hydrogen liquid state [10]. However, more detailed studies indicate it to be a solidphase structure where randomly distributed $\mathrm{H}_{2}$ molecules are sandwiched between graphene-like sheets of atomic hydrogen [12-16].

A schematic phase diagram of hydrogen is illustrated in figure 1. Figure 1 shows that hydrogen would be in atomic liquid state at a pressure of $450 \mathrm{GPa}$ and a temperature of $300 \mathrm{~K}$. In this paper, we perform molecular dynamics (MD) simulations to investigate the effect of nuclear quantum effects on the pair correlations of $\mathrm{H}$ atoms in the liquid state at room temperature and a pressure of $450 \mathrm{GPa}$. The calculated paircorrelation functions show that the nuclear quantum effects are quite significant for the correct description of liquid atomic hydrogen at high pressures.

\section{Computational details}

The electronic structure calculations are performed using the density functional theory and the projector augmentedwave (PAW) method [17] as implemented in Quantum Espresso package [18]. The exchange-correlation interactions are represented by using the Perdew-Burke-Ernzerhof (PBE) type of generalized gradient approximation (GGA) 


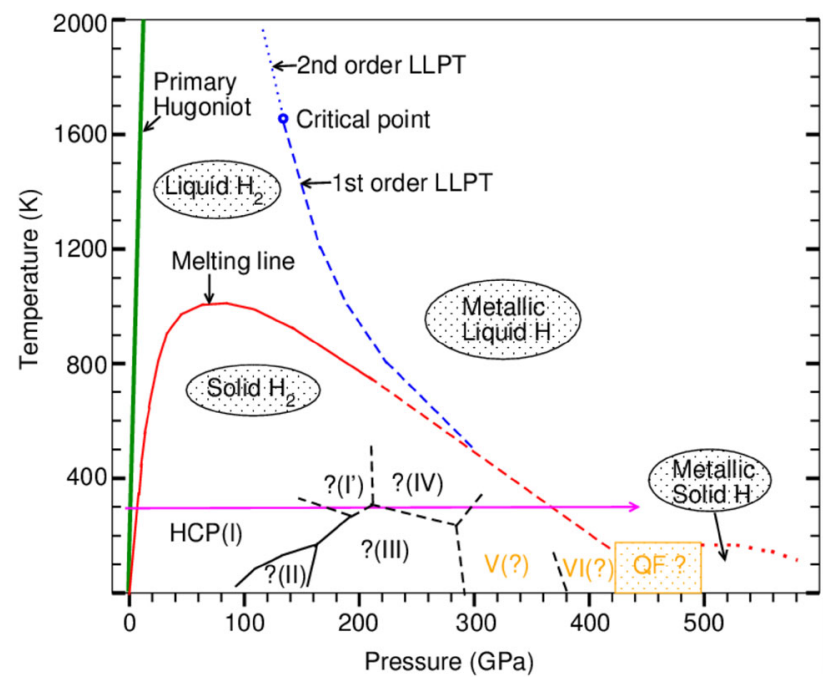

Figure 1. Schematic phase diagram of hydrogen. The solid lines represent the experimentally confirmed transitions between different phases of hydrogen, while the dotted lines represent the theoretically predicted transitions. This phase diagram shows three types of transitions; (1) the transition between liquid and solid states of hydrogen, (2) the transition between molecular and atomic hydrogen, (3) the transition between different phases of solid hydrogen. At a pressure of $450 \mathrm{GPa}$ and a temperature of $300 \mathrm{~K}$, the hydrogen is expected to be in an atomic liquid state.

[19]. We first performed the convergence calculations on the hydrogen molecule for energy cutoff on the plane wave basis set for the expansion of a wavefunction required for the electronic structure calculations. The results using the Coulomb potential and PAW pseudo-potential are shown in figure 2. At about $60 \mathrm{Ry}$ of energy cutoff the total energy and the bond length of hydrogen molecule get converged for the PAW pseudo-potential. It means that the energy cutoff of $60 \mathrm{Ry}$ is sufficient to perform electronic calculations of a hydrogen system using PAW pseudopotential.

We considered energy cutoff of 60 Ry for further electronic structure calculations of the hydrogen system. We construct cubic hydrogen supercells of 128 and $216 \mathrm{H}$ atoms placed randomly within the supercell provided the distance between the $\mathrm{H}$ atoms are greater than $1 \AA$. We then perform classical BornOppenheimer molecular dynamic (BOMD) simulations in isobaric-isothermal (NPT) and canonical (NVT) ensembles using a time step of 8 a.u. (in Rydberg atomic units 1 a.u. $=0.048378 \mathrm{fs}$ ). We simulate supercells for about $20 \mathrm{ps}$. We also include the nuclear quantum effects in 128 atom supercells using the path integral formulation of quantum mechanics (PIMD) [20]. In this approach, each quantum particle is represented by $\mathrm{P}$ quasi-beads, labelled as 1 to $\mathrm{P}$. During the simulation, each quasi-bead interacts with its nextnearest neighbour quasi-beads through the spring potential in imaginary-time scale.
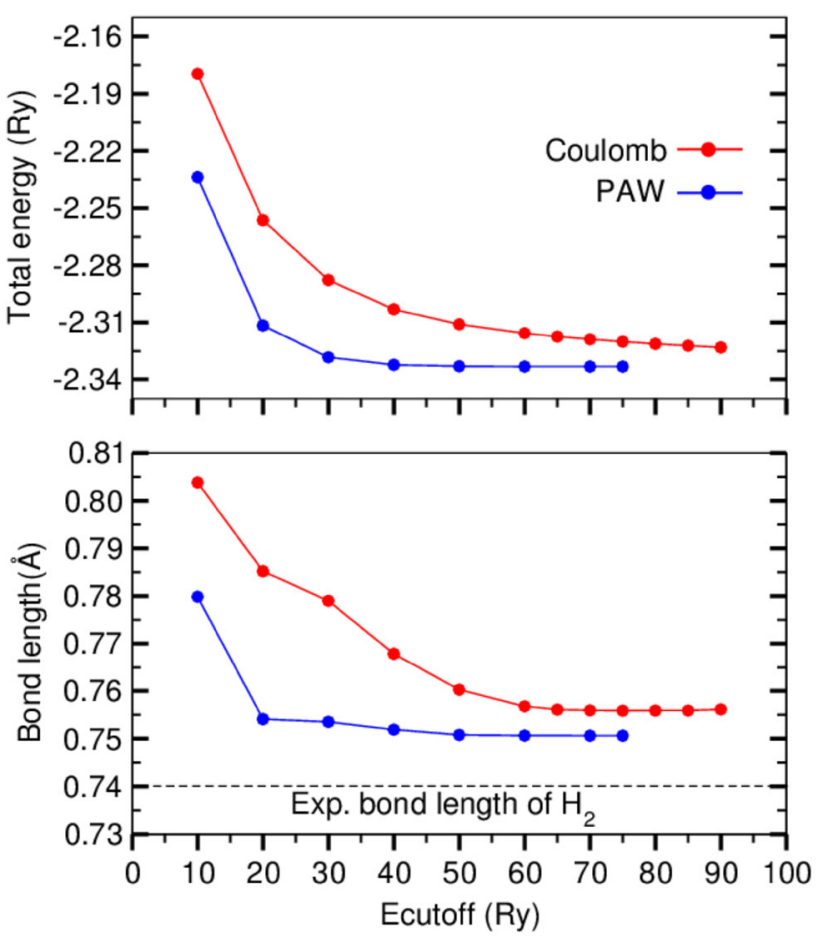

Figure 2. Convergence tests on $\mathrm{H}_{2}$ molecule for total energy and bond length using the Coulomb and PAW pseudo-potentials. The total energy and bond length of $\mathrm{H}_{2}$ molecule get converged at the cutoff energy of 60 Ry for PAW pseudo-potentials.

\section{Results and discussion}

We first simulate the supercells at temperatures of 200 $500 \mathrm{~K}$ and a pressure of $450 \mathrm{GPa}$ using BOMD simulations. After initial thermal equilibration for 10,000 simulation time steps, we take equidistantly spaced snapshot atomic configurations. The pair correlations of atoms in the simulated supercells of MD simulations are indicators of structural correlations of atoms in the supercell. We have calculated pair-correlation functions as ensemble averages of 100 equidistantly selected atomic configurations from our MD simulations. The calculated pair-correlation functions are given in figures 3 and 4 .

Figure 3 shows the pair-correlation functions of $\mathrm{H}$ atoms in the simulated supercells of 216 atoms within NVT ensembles at a pressure of $490 \mathrm{GPa}$ and temperatures of 200-500 K. There are prominent peaks in the pair-correlation functions. These peaks indicate that there are the $\mathrm{H}_{2}$ molecular formations in the supercells. The height of these peaks decreases with increasing temperatures, which means $\mathrm{H}_{2}$ molecular correlations decrease with increasing temperature. At $500 \mathrm{~K}$ and $490 \mathrm{GPa}$, the pair-correlation function shows that the simulated $\mathrm{H}$ system is still not in the atomic hydrogen liquid state. However, the phase diagram in figure 1 indicates that at $300 \mathrm{~K}$ and $490 \mathrm{GPa}$, the $\mathrm{H}$ system should be in the atomic liquid state. This means either the phase diagram is wrong or BOMD simulations are not accurate enough to simulate $\mathrm{H}$ 


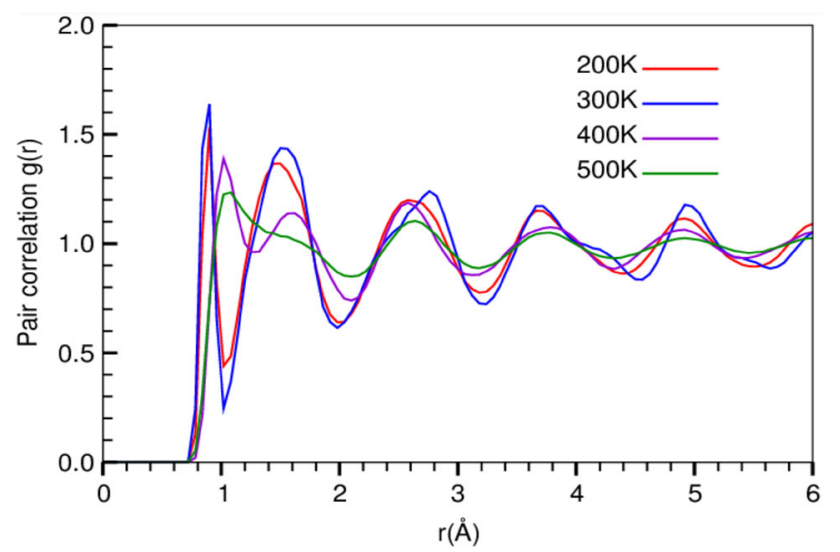

Figure 3. Pair-correlation function of liquid hydrogen under BOMD simulations at different temperatures within NVT ensemble. The pair-correlation functions show that there is $\mathrm{H}_{2}$ molecular structural formations in BOMD simulated hydrogen systems. These molecular structural formations decrease with increasing temperature. These pair-correlation functions indicate that the simulated hydrogen system under BOMD simulations have significant molecular $\mathrm{H}_{2}$.

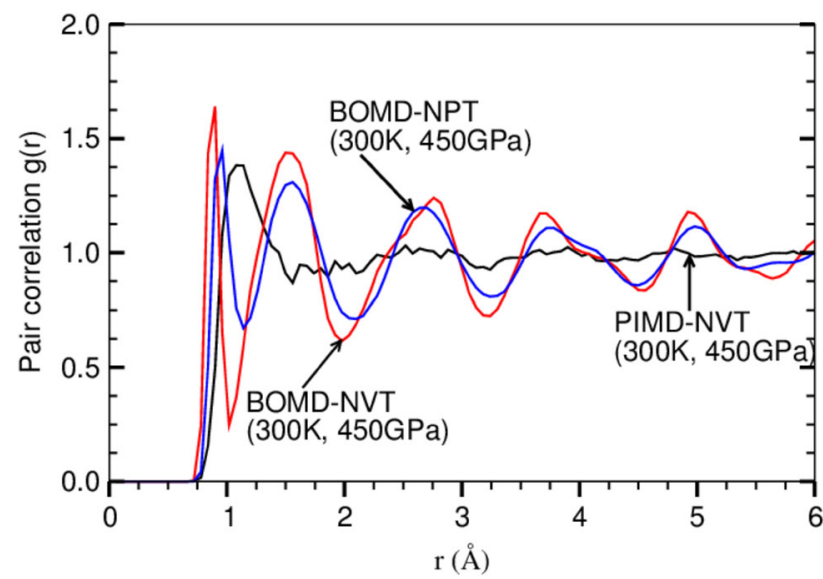

Figure 4. Pair-correlation function of liquid hydrogen under BOMD and PIMD simulations. The pair-correlation function shows that there is $\mathrm{H}_{2}$ molecular structural formations in BOMD simulated hydrogen systems within NPT as well as NVT ensembles. However, in PIMD simulations there are no molecular structural formations in the hydrogen system. Thus, the nuclear quantum effects are quite crucial for the correct description of atomic hydrogen at room temperature and a pressure of $490 \mathrm{GPa}$.

systems. To check this we include nuclear quantum effects in the MD simulations using the quasi-beads simulation approach.
Figure 4 shows the pair-correlation functions of $\mathrm{H}$ atoms at $300 \mathrm{~K}$ and $450 \mathrm{GPa}$ of simulated supercells with BOMD and PIMD approach. We used 24 beads for each atom to include the nuclear quantum effects of $\mathrm{H}$ atoms in the supercell of 128 atoms. The BOMD simulated systems show that the $\mathrm{H}$ system is in molecular liquid state. However, the paircorrelation function of $\mathrm{H}$ atoms with inclusion of nuclear quantum effects using the PIMD shows that the $\mathrm{H}$ system is in atomic liquid state at room temperature and a pressure of $490 \mathrm{GPa}$.

In summary, we simulated the hydrogen cubic supercells of 216 and 128 atoms at room temperature and a pressure of $490 \mathrm{GPa}$. We simulated these supercells within the BOMD and PIMD simulations. After thermal equilibrium for some time, we took snapshot configurations for calculations of pair-correlation functions of $\mathrm{H}$ atoms. The calculated paircorrelation function shows that the nuclear quantum effects are quite significant for the correct description of liquid hydrogen.

\section{References}

[1] Dattagupta S 1996 Bull. Mater. Sci. 1915

[2] Agarwal S C 1996 Bull. Mater. Sci. 1939

[3] Ambat R and Dwarakadasa E S 1996 Bull. Mater. Sci. 19103

[4] Simsek M 2017 Bull. Mater. Sci. 40907

[5] Gomez Costa M B, Juaraz J M, Pecchi G and Anunziata O A 2017 Bull. Mater. Sci. 40271

[6] Mao H K and Hemley R J 1994 Rev. Mod. Phys. 66671

[7] Weir S T, Mitchell A C and Nellis W J 1996 Phys. Rev. Lett. 761860

[8] Johnson K A and Ashcroft N W 2000 Nature 403632

[9] Wigner E and Huntington H B 1935 J. Chem. Phys. 3764

[10] Eremets M I and Troyan I A 2011 Nat. Mater. 10927

[11] Zha C S, Cohen R E, Mao H K and Hemley R J 2014 Proc. Natl. Acad. Sci. 1104792

[12] Pickard C J, Martinez-Canales M and Needs R J 2012 Phys. Rev. B 85214114

[13] Liu H, Wang H and Ma Y 2012 J. Phys. Chem. C 1169221

[14] Labet V, Hoffmann R and Ashcroft N W 2012 J. Chem. Phys. 136074504

[15] Liu H and Ma Y 2013 Phys. Rev. Lett. 110025903

[16] Goncharov A F, Tse J S, Wang H, Yang J, Struzhkin V V, Howie R T et al 2013 Phys. Rev. B 87024101

[17] Blöchl P E 1994 Phys. Rev. B 5017953

[18] Giannozzi P, Baroni S, Bonini N, Calandra M, Car R, Cavazzoni C et al 2009 J. Phys.: Condens. Matter 21395502

[19] Perdew J P, Burke K and Ernzerhof M 1996 Phys. Rev. Lett. 77 3865

[20] Ceriotti M, More J and Manolopoulos D E 2014 Comp. Phys. Commun. 1851019 